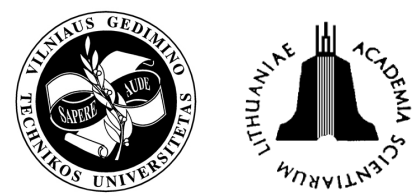

\title{
DETERMINATION OF TRAFFIC FLOW PARAMETERS IN DIFFERENT TRAFFIC FLOW INTERACTION CASES
}

\author{
Raimundas Junevičius ${ }^{1}$, Marijonas Bogdevičius ${ }^{2}$ \\ Dept of Transport Technological Equipment, Vilnius Gediminas Technical University, Plytinès g. 27 \\ LT-10105 Vilnius, Lithuania. Phone +370 52744782 \\ E-mails: ${ }^{1}$ rj@ti.vgtu.lt; ${ }^{2}$ marius@ti.vgtu.lt
}

Received 4 January 2007; accepted 2 May 2007

\begin{abstract}
Modelling of straight road section consisting of one traffic line gives the opportunity to simulate "follow the car" system. In general it looks like a line of vehicles, going one after another. Kinetic theory, used in this paper describes traffic flow system as a straight unbroken line with limited flow speed and concentration. Such model also gives the opportunity to derive traffic lines intersections. For example, intersection could be derived like a point with traffic lines coming and outgoing from this point by only changing boundary conditions. Mathematical model is built using characteristic method.
\end{abstract}

Keywords: Traffic flow, kinetic theory, modelling, characteristic method.

\section{Introduction}

Traffic lines intersection gives difficulties to model two-way crossroads. To solve this problem at first step we are modelling one-way one line traffic flow intersection with two incoming one outgoing and with one incoming and two outgoing traffic flows. Such model gives solution to classic bottleneck situation on a road. The second step is to determine two line traffic flow model to solve various crossroad problems.

Similar situations were studied in literature [1-6]. Authors in the works $[1,2]$ based their solutions on kinetic theory, made various traffic flow models on straight road, driver behaviour model. Authors of literature [3] did research into traffic modelling and control using neural networks, authors $[6,7]$ concentrated on traffic control and regulations in cities.

In this paper at first we derive equations of traffic flow on straight road using characteristic method, and using these results determine traffic flow intersections.

\section{Traffic flow model using characteristic method}

Basic equations of traffic flow model using kinetic theory are [3]:

$$
\frac{\partial k}{\partial t}+V \frac{\partial k}{\partial x}+k \frac{\partial V}{\partial x}=0
$$

$$
k \frac{\partial V}{\partial t}+k V \frac{\partial V}{\partial x}+\Theta \frac{\partial k}{\partial x}=k\left\langle\frac{d v}{d t}\right\rangle_{v}-k \frac{\partial \Theta}{\partial x}
$$

where: $k$ - concentration; $V$ - traffic flow speed; $\Theta-$ speed variation; $x$ - point on a road; $v$ - vehicle speed.

Using characteristic method the road section is divided into pieces of length $\Delta x$ (Fig 1) [5]. Each piece has information which is concentrated in boundaries. Each peace boundary point takes information: velocity $v$ and concentration $k$.

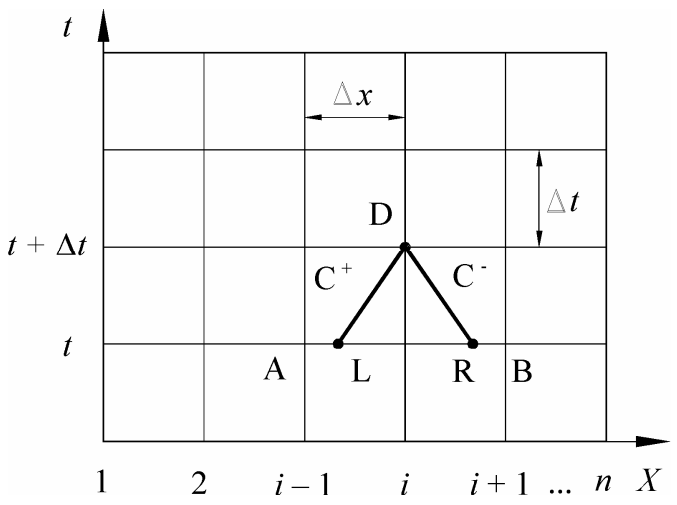

Fig 1. Characteristic net

System of equations (1) and (2) can be written as the system of second-order quasi-linear differential equations: 


$$
\left.\left.[A]\left\{\frac{\partial u}{\partial t}\right\}+[B]\right\} \frac{\partial u}{\partial x}\right\}=\{f\}
$$

where: $[A],[B]$ are matrices,

$$
[A]=\left[\begin{array}{ll}
1 & 0 \\
0 & k
\end{array}\right] ;[B]=\left[\begin{array}{cc}
V & k \\
\Theta & k V
\end{array}\right]
$$

and $\{f\}$ is vector,

$$
\{f\}=\left\{k\left\langle\frac{d v}{d t}\right\rangle_{v}^{0}-k \frac{\partial \Theta}{\partial x}\right\}
$$

which depends on $t, x$ and elements $u_{i}$ of vector $\{u\}^{T}=$ $[k, V]$.

Equating the determinant of matrix $[B]-[A] \frac{d x}{d t}$ to zero,

$$
\left|[B]-[A] \frac{d x}{d t}\right|=0
$$

we shall receive the equation which allows determining $\frac{d x}{d t}$ derivative and determines characteristic direction.

This equation has two various real roots $d x / d t=$ $\lambda_{i}(i=1,2)$ :

$$
\begin{aligned}
& \mathrm{C}^{+}: \frac{d x}{d t}=V+\sqrt{\Theta} ; \\
& \mathrm{C}^{-}: \frac{d x}{d t}=V-\sqrt{\Theta},
\end{aligned}
$$

From equations (1) and (2), using steps listed above, after several transformations receive plus and minus characteristics which have such forms:

$$
\begin{aligned}
& \mathrm{C}^{+}: \frac{d V}{d t}+\frac{\sqrt{\Theta}}{k} \frac{d k}{d t}-g_{1}=0 ; \\
& \mathrm{C}^{-}: \frac{d V}{d t}-\frac{\sqrt{\Theta}}{k} \frac{d k}{d t}-g_{2}=0,
\end{aligned}
$$

where $g_{i}(i=1,2)$ are traffic flow speed variations.

$$
\begin{aligned}
& g_{1}=\left\langle\frac{d v}{d t}\right\rangle_{v}-\frac{\partial \Theta}{\partial x} \\
& g_{2}=\left\langle\frac{d v}{d t}\right\rangle_{v}-\frac{\partial \Theta}{\partial x} .
\end{aligned}
$$

The system of two nonlinear algebraic equations is obtained from conditions of compatibility with characteristics:

$$
\begin{aligned}
& \Phi_{1}=V_{D}-V_{L}+\frac{1}{2}\left[\left(\frac{\sqrt{\Theta}}{k}\right)_{L}+\left(\frac{\sqrt{\Theta}}{k}\right)_{D}\right] \times \\
& \left(k_{D}-k_{L}\right)-\frac{\Delta t}{2}\left(g_{1 L}+g_{1 D}\right)=0
\end{aligned}
$$

$$
\begin{aligned}
& \Phi_{2}=V_{D}-V_{R}-\frac{1}{2}\left[\left(\frac{\sqrt{\Theta}}{k}\right)_{R}+\left(\frac{\sqrt{\Theta}}{k}\right)_{D}\right] \times \\
& \left(k_{D}-k_{R}\right)-\frac{\Delta t}{2}\left(g_{2 D}+g_{2 R}\right)=0 ;
\end{aligned}
$$

From these equations receive two parameters $\mathrm{v}$ and $\mathrm{k}$ in each road section boundary point. To determine traffic flow value in road section at first it is needful to determine average flow speed. Generally it could be average vehicle speed.

Equation to calculate traffic flow $q$ has the following form [1]:

$$
q=k V \text {. }
$$

Equations listed above could be used for solving different traffic flow problems.

\section{Traffic flow intersections}

In this chapter three typical intersections schemes will be determined. The first one is a situation when two different traffic flows come into one, as shown in Fig 2.

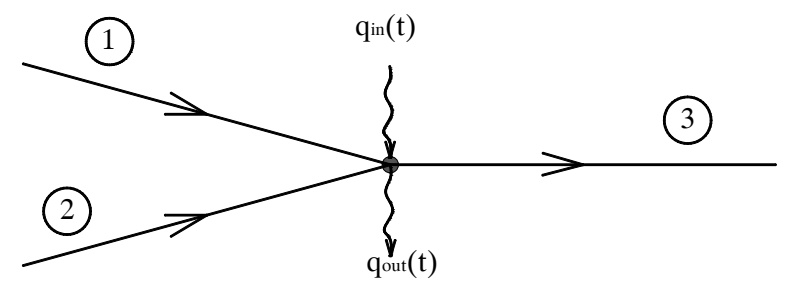

Fig 2. Traffic flow intersection diagram

Intersection is determined as a point at which three separate flows cross. In this case to model interflow two $\mathrm{C}^{+}$and one $\mathrm{C}^{-}$characteristics and additionally one input and one output leaks are used. Equations of this model could be written in the following form:

$$
\begin{gathered}
\mathrm{C}^{+}: \Phi_{1}=V_{D, 1}-V_{L, 1}+\frac{1}{2}\left[\left(\frac{\sqrt{\Theta}}{k}\right)_{L, 1}+\left(\frac{\sqrt{\Theta}}{k}\right)_{D, 1}\right] \times \\
\left(k_{D, 1}-k_{L, 1}\right)-\frac{\Delta t}{2}\left(g_{1 L, 1}+g_{1 D, 1}\right)=0 ; \\
\mathrm{C}^{+}: \Phi_{2}=V_{D, 2}-V_{L, 2}+\frac{1}{2}\left[\left(\frac{\sqrt{\Theta}}{k}\right)_{L, 2}+\left(\frac{\sqrt{\Theta}}{k}\right)_{D 2}\right] \times \\
\left(k_{D, 2}-k_{L, 2}\right)-\frac{\Delta t}{2}\left(g_{1 L, 2}+g_{1 D, 2}\right)=0 ; \\
\mathrm{C}^{-}: \Phi_{3}=V_{D, 3}-V_{R, 3}-\frac{1}{2}\left[\left(\frac{\sqrt{\Theta}}{k}\right)_{R, 3}+\left(\frac{\sqrt{\Theta}}{k}\right)_{D, 3}\right] \times \\
\left(k_{D, 3}-k_{R, 3}\right)-\frac{\Delta t}{2}\left(g_{2 D, 3}+g_{2 R, 3}\right)=0 .
\end{gathered}
$$

Then traffic flow becomes: 


$$
\begin{aligned}
& p_{1}(k V)_{D, 1}-p_{2}(k V)_{D, 2}-p_{3}(k V)_{D, 3}- \\
& p_{4} q_{0 u t}(t)+p_{5} q_{\text {in }}(t)=0, \\
& \sum_{i=3}^{5} p_{i}=1,
\end{aligned}
$$

here: $p_{i}$ - probability that action may be done.

The second one is a situation when single traffic flow splits into two different traffic flows (Fig 3).

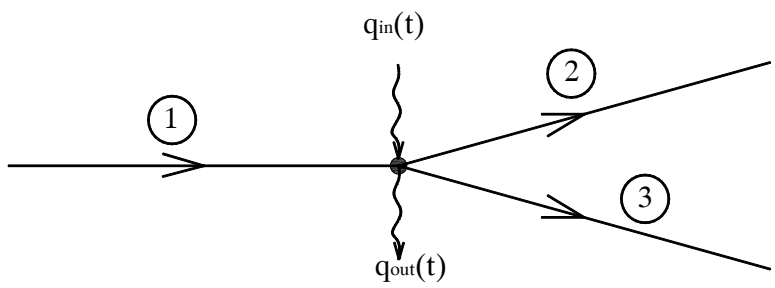

Fig 3. Traffic flow intersection diagram

Intersection also is determined as a point at which three separate flows cross. In this case one $\mathrm{C}^{+}$and two $\mathrm{C}^{-}$characteristics and additionally one input and one output leaks are used. Equations of this model could be written in the following form:

$$
\begin{gathered}
\mathrm{C}^{+}: \Phi_{1}=V_{D, 1}-V_{L, 1}+\frac{1}{2}\left[\left(\frac{\sqrt{\Theta}}{k}\right)_{L, 1}+\left(\frac{\sqrt{\Theta}}{k}\right)_{D, 1}\right] \times \\
\left(k_{D, 1}-k_{L, 1}\right)-\frac{\Delta t}{2}\left(g_{1 L, 1}+g_{1 D, 1}\right)=0 ; \\
\mathrm{C}^{-}: \Phi_{2}=V_{D, 2}-V_{R, 3}-\frac{1}{2}\left[\left(\frac{\sqrt{\Theta}}{k}\right)_{R, 2}+\left(\frac{\sqrt{\Theta}}{k}\right)_{D, 2}\right] \times \\
\left(k_{D, 2}-k_{R, 2}\right)-\frac{\Delta t}{2}\left(g_{2 D, 2}+g_{2 R, 2}\right)=0 ; \quad(17) \\
\mathrm{C}^{-}: \Phi_{3}=V_{D, 3}-V_{R, 3}-\frac{1}{2}\left[\left(\frac{\sqrt{\Theta}}{k}\right)_{R, 3}+\left(\frac{\sqrt{\Theta}}{k}\right)_{D, 3}\right] \times \\
\left(k_{D, 3}-k_{R, 3}\right)-\frac{\Delta t}{2}\left(g_{2 D, 3}+g_{2 R, 3}\right)=0 .
\end{gathered}
$$

Then traffic flow becomes:

$$
\begin{aligned}
& p_{1}(k V)_{D, 1}-p_{2}(k V)_{D, 2}-p_{3}(k V)_{D, 3}- \\
& p_{4} q_{0 u t}(t)+p_{5} q_{\text {in }}(t)=0 ; \\
& \sum_{i=2}^{5} p_{i}=1 .
\end{aligned}
$$

The third one is intersection of four roads. Each road has two, opposite direction of movement, traffic lines (Fig 4).

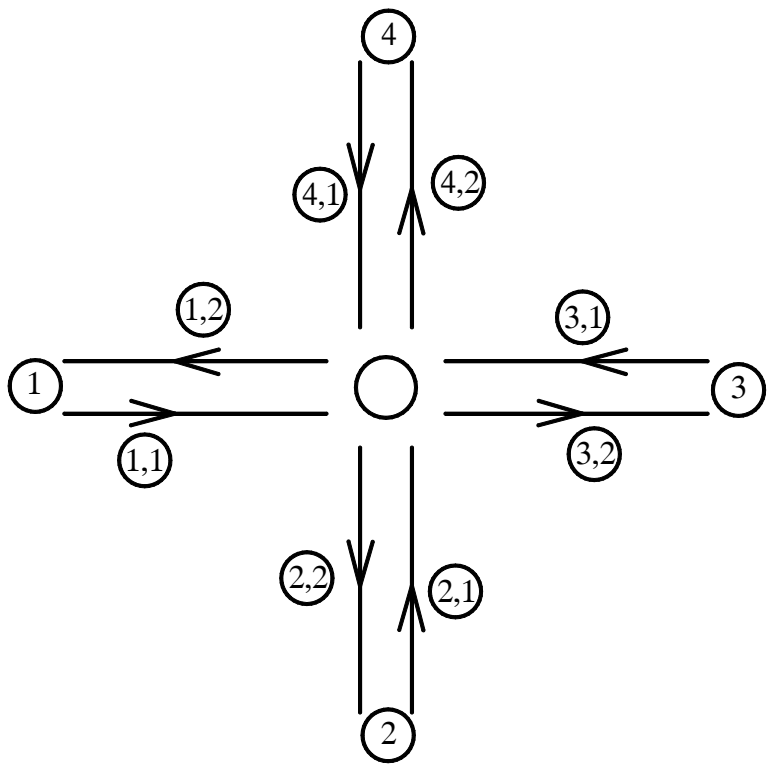

Fig 4. Traffic flow intersection diagram

In this case to determine equations of movement on each road line it is needful to assign $\mathrm{C}^{+}$characteristic on outgoing traffic line and $\mathrm{C}^{-}$characteristic on ingoing traffic line. Boundary condition of each road at intersecting point is built using equations (8) and (9). In primitive form these equations can be written in the following way:

$$
\left\{\begin{array}{l}
K, 1: C^{+}: \Phi_{1}=\Phi_{1}\left(V_{D, k, 1}, k_{D, k, 1}\right)=0 ; \\
K, 2: C^{-}: \Phi_{2}=\Phi_{2}\left(V_{D, k, 2}, k_{D, k, 2}\right)=0 .
\end{array}\right.
$$

In this equation index 1 is used for incoming and index 2 is used for outgoing traffic flows.

Each of four roads is derived in the same order so in the end receiving equation system from 8 equations.

When boundary conditions are set, the next step is to balance incoming and outgoing flows. Traffic flows balance derivation then takes the following form:

$$
\Phi_{9}=\sum_{k=1}^{4} p_{k, 1}(k V)_{k, 1}-\sum_{k=1}^{4} p_{k, 2}(k V)_{k, 2}=0 .
$$

Now there are all 9 equations and it is almost possible to get a solution. The final step to derive equations is to set correct probability laws for all traffic lines.

Probability equation for incoming flow in general form is expressed as:

$$
\begin{aligned}
& p_{1,1}+p_{1,2,2}+p_{1,3,2}+p_{1,4,2}=1 ; \\
& p_{2,1}+p_{2,2,2}+p_{2,3,2}+p_{2,4,2}=1 ; \\
& p_{3,1}+p_{3,2,2}+p_{3,3,2}+p_{3,4,2}=1 ; \\
& p_{4,1}+p_{4,2,2}+p_{4,3,2}+p_{4,4,2}=1 ; \\
& p_{k, 1}+\sum_{i \check{1} 1}^{4} p_{k, i, 2}=1 ; i \neq k .
\end{aligned}
$$


Probability equation for outgoing flow in general form will be as follows:

$$
\begin{aligned}
& p_{1,2,2}+p_{3,2,2}+p_{4,2,2}=1 ; \\
& p_{1,3,2}+p_{2,3,2}+p_{4,3,2}=1 ; \\
& p_{1,4,2}+p_{2,4,2}+p_{3,4,2}=1 ; \\
& p_{2,1,2}+p_{3,1,2}+p_{4,1,2}=1 ; \\
& \sum_{i=1}^{4} p_{k, i, 2}=1 ; i=1, . ., 4 ; i \neq k .
\end{aligned}
$$

Equations (23) and (24) show that incoming flow from any of four roads can be splited and steered to all three, to two or one outgoing lines. This could be done by changing probability values from 0 to 1 . Probability can be taken like the opportunity of choice. Then model may be reorganized so as to minimise or maximise the desired parameter. Or probability may be taken as a strict rule and in that case it is possible to model traffic-light controlled crossroads.

Solution of such equations has quite a simple form. In general it can be as follows:

$$
\left\{\begin{array}{l}
k_{i}^{t+\Delta t}=k_{i}^{t}+d k \\
v_{i}^{t+\Delta t}=v_{i}^{t}+d v .
\end{array}\right.
$$

where:

$$
\left\{\begin{array}{l}
d k \\
d v
\end{array}\right\}=-\left[\begin{array}{l}
\frac{\partial \Phi_{1}}{\partial k} \frac{\partial \Phi_{1}}{\partial v} \\
\frac{\partial \Phi_{2}}{\partial k} \frac{\partial \Phi_{2}}{\partial v}
\end{array}\right]^{-1}\left\{\begin{array}{l}
\Phi_{1} \\
\Phi_{2}
\end{array}\right\}
$$

These equation systems are used to derive the solution for each section boundary point. To get a solution it is very important to take correct time step of calculation $\Delta t$. Time interval $\Delta t$ is related to road section $\Delta x$. To get the right solution it is important to sustain the specification [5]:

$$
C r=\frac{\Delta t}{\Delta x} \leq 1 .
$$

In the end, such or similar model could be used to solve problems in various road intersection cases, in uncontrolled traffic flows, and to solve problems related to traffic control in cities.

\section{Conclusions}

1. Using characteristic method and kinetic theory it is possible to make traffic line intersection models in quite a simple way and the models are suitable to use for solving various problems.

2. Intersection models in general are similar to other models, but in this case it is possible to determine the dynamic system of long straight road sections intersecting each other.

\section{References}

1. PRIGOGINE, I.; HERMAN, R. Kinetic theory of vehicular traffic. New York, 1971. $101 \mathrm{p}$.

2. TAMPÈRE, C. Human-kinetic multiclass traffic flow theory and modelling with application to advanced driver assistance systems in congestion, T2004/11, TRAIL Research School. Netherlands, 2004. 333 p.

3. Traffic flow modelling and control using Artifical Neural Networks, 0272-1708/96/\$05.000 1996 IEEE.

4. HELBING, D.; GREINER, A. Modelling and simulation of multi-lane traffic flow. Phys. Rev. E 55, 1999, p. 54985508.

5. BOGDEVICIUS, M.; PRENTKOVSKIS, O. Dynamics of hidraulic and pneumatic systems (Hidrauliniu ir pneumatiniu sistemų dinamika). Vilnius: Technika, 2003. 264 p. (in Lithuanian).

6. SILJANOV, V. V. Traffic flow theory in road design and traffic control (Теория транспортных потоков в проектировании дорог и организации движения). Moscow, 1977. 304 p. (in Russian).

7. INOSE, X; XAMADA, T. Road traffic control (Управление дорожным движением). Moscow, 1983. 248 p. (in Russian). 